\title{
Distributional regression analysis of learning analytics and educational data
}

\author{
Fernando Marmolejo-Ramos · Mauricio Tejo · \\ Marek Brabec · Jakub Kuzilek · Srecko \\ Joksimovic · Vitomir Kovanovic · Jorge González · \\ Raydonal Ospina
}

Received: date / Accepted: date

\begin{abstract}
The advent of technological developments is allowing gathering large amounts of data in several research fields. Learning analytics/educational data mining (LA/EDM) has access to big observational unstructured data captured from educational settings and relies mostly on unsupervised machine learning algorithms to make sense of such type of data. Generalised additive models of location, scale and shape (GAMLSS) are supervised statistical learning approaches that allow modelling all the parameters of the distribution of the response variable w.r.t. the explanatory variables. This article briefly introduces the power
\end{abstract}

Fernando Marmolejo-Ramos

Centre for Change and Complexity in Learning, University of South Australia, Adelaide, Australia

E-mail: fernando.marmolejo-ramos@unisa.edu.au

Mauricio Tejo

Departamento de Matemática, Universidad Tecnológica Metropolitana, Chile

E-mail:m.tejoa@utem.cl

Marek Brabec

Department of Statistical Modelling, Institute of Computer Science of the Czech Academy of Sciences, Prague, Czech Republic

E-mail: mrabec@cs.cas.cz

Jakub Kuzilek

Czech Institute of Informatics, Robotics and Cybernetics, CTU in Prague; and

Computer Science Education/Computer Science and Society research group, Humboldt University of Berlin E-mail: jakub.kuzilek@hu-berlin.de

Srecko Joksimovic

Centre for Change and Complexity in Learning, University of South Australia, Adelaide, Australia E-mail: srecko.joksimovic@unisa.edu.au

Vitomir Kovanovic

Centre for Change and Complexity in Learning, University of South Australia, Adelaide, Australia

E-mail: vitomir.kovanovic@unisa.edu.au

Jorge González

Departamento de Estadística, Pontificia Universidad Católica de Chile, Santiago de Chile, Chile

E-mail: jorge.gonzalez@mat.uc.cl

Raydonal Ospina

Department of Statistics, CASTLab, Federal University of Pernambuco, Recife, Brazil

E-mail: raydonal@de.ufpe.br 
and flexibility of GAMLSS to the LA/EDM community in order to prompt a distributional and interpretable statistical learning of data.

Keywords supervised learning · statistical learning · learning analytics · educational data mining $\cdot$ generalised additive models of location, scale, and shape $\cdot$ statistical modelling

\section{Introduction}

Most of the data in the field of learning analytics (LA) and educational data mining (EDM) are characterised by being big, second-hand, observational, and unstructured (e.g. Motz et al. (2018) 1 The data are big because they come from physical and virtual educational environments with many instructors and thousands of students and for whom several metrics exist (e.g. number of clicks, time stamps, course grades, etc). The data are second-hand, observational, and unstructured because they are not obtained directly and the type and number of variables are not controlled by the researcher. Although such type of data are amenable to post-hoc analyses only and do not allow confident causal inference (i.e. only experimentation enables so by securing first-hand and structured data; see Imai et al. (2008)), they are nonetheless rich and should be statistically treated to extract valuable practical information 2

After gathering educational data, the LA/EDM analytical pipeline begins with preprocessing the data so it is amenable to subsequent statistical treatment. Data pre-processing consumes more than $50 \%$ of the pipeline and, among other things, it implies selecting and transforming variables of interest (Romero and Ventura (2020)). Given a large chunk of the analytical pipeline is spent on data pre-processing, it is no surprise that unsupervised learning algorithms are heavily relied on in order to make sense of the data (Joksimovic et al. (2018)). Indeed, advances in machine learning (ML) enable to submit data to a set of algorithms that have the same goal (e.g. classification algorithms) via ensemble learning (e.g. targeted learning [a.k.a., superlearner]; see Van der Laan (2017)). The appeal of those algorithms is that they allow finding patterns in data with minimal human supervision.

Once an explanatory (unsupervised) model has been identified, it is tested for its predictive power (e.g. via cross-validation; see Yu and Kumbier (2020) for a proposal of the place of cross-validation in the analytic pipeline). Although unsupervised learning algorithms can meet their intended goal, their usage implies moving away from proper statistical learning or modelling. In other words, unsupervised learning algorithms minimise human decisionmaking in the process of statistical model building. This article has the goal of introducing generalised additive models of location, scale and shape (GAMLSS); a supervised learning regression method that promotes attending to statistical modelling by requiring human supervision. The outline of this article is as follows: first, a brief technical description of GAMLSS is provided; second, an LA data set is described; and third, GAMLSS modelling is performed on the data. The discussion section considers distributional modelling in the larger context of statistical learning.

\footnotetext{
${ }^{1}$ In this article, LA and EDM are referred to indistinctly.

2 An example of estimating causality with observational data is found in Bates et al. 2020); a brief commentary of that study can be found in Bühlmann (2020b).
} 


\section{GAMLSS as a distributional regression framework for statistical learning}

One of the traditional pre-processing practices in LA/EDM research consists of discretising continuous variables in order to enhance their interpretability (see section 3.3 in Romero and Ventura (2020)). Slicing an uniform or normally distributed continuous variable in three quantile-based bins (i.e. high, medium, and low) has been shown to approximate quite well a linear regression (Gelman and Park (2008)). However, in practice, and particularly in the social sciences and education fields, continuous variables tend to follow non-normal shapes (Bono et al. (2017)). This fact then suggests that traditional regression models are not optimal and slicing numeric variables will give biased results (see Bennette and Vickers (2012) for an example of how categorisation of continuous data in epidemiology leads to biased estimation). Hence, flexible and interpretable regression techniques are needed to model such type of data. GAMLSS is a regression framework that enables performing comprehensive statistical learning on the distribution of the response variable w.r.t. the covariates.

GAMLSS are a class of supervised learning tools for semi-parametric regression problems and that have led to a growing sophistication in the ML field. From an strict statistical modelling viewpoint (McCullagh, 2002), GAMLSS are used to analyse nonlinear relationships between the distributions of outcomes and features (covariates) and where the feature effects are additively weighted. These models were proposed by Rigby and Stasinopoulos (2001); Akantziliotou et al. (2002) and (Rigby and Stasinopoulos 2005) as an improvement and extension to the generalised linear models (GLM) (Nelder and Wedderburn 1972; McCulloch, 2000) and the generalised additive models (GAM) (Hastie and Tibshirani 1990). Key to GAMLSS is that they enable data analyses that exhibit parsimony, generality, consilience and predictive capacity (Friedman and Silverman, 1989, Picard and Cook, 1984).

GAMLSS have been used in several fields including ML (Hofner et al. 2014, Groll et al., 2019, Mayr et al. 2012), ecology (Smith et al., 2019), survival analysis (De Castro et al. 2010); clinical management of hearing loss (Hu et al. 2015), insurance (Gilchrist et al. 2009), real state appraisal of land lots (Florencio et al. 2012), among others. Softwarewise, GAMLSS is implemented in R through the gamlss package (Stasinopoulos et al. 2007, 2017, Rigby et al. 2020). There are other GAMLSS R packages for extra additive terms (gamlss.add), fitting censored (interval) responses (gamlss.add), fitting finite mixture distributions (gamlss.mx), fitting non-linear models (gamlss.nl), fitting truncated distributions (gamlss.tr), among others. Other R packages related to GAMLSS are gamboostLSS and BAMLSS and these allow performing boosting methods for GAMLSS models and Bayesian GAMLSS, respectively (Thomas et al., 2018; Umlauf et al., 2018, De Bastiani et al. 2018).

Another appealing feature of GAMLSS is its flexibility for data modelling through estimation algorithms (Cole and Green, 1992, Rigby and Stasinopoulos, 1996) that allow combining ML with statistical modelling (Stasinopoulos et al., 2018, Breiman, 2001). For example, such algorithms enable fitting the conditional parametric distribution of the response variable with several continuous, discrete and mixed distributions with different degrees of asymmetry and kurtosis. Therefore, not only the mean, but all of the parameters (i.e. location, scale and shape) can be modelled as parametric and/or additive non-parametric functions of covariates. This feature is quite instrumental to modelling response variables that do not follow an exponential family distribution (some exponential distributions are the Normal, Poisson, Gamma, Beta, Weibull and Multinomial distributions (Casella and Berger. 2002, McCulloch, 2000, Barndorff-Nielsen, 1980)).

More specifically, consider a dataset $\left(\mathbf{X}_{k}, \mathbf{Z}_{k}, \mathbf{y}\right)_{k \leq p}$ of sample size $n$, where $\mathbf{y}=\left(y_{1}, y_{2}, \ldots, y_{n}\right)^{\top}$ is a vector of independent observations on the response variable and $\mathbf{X}_{k}, \mathbf{Z}_{k}$ are input co- 
variates design matrices for fixed and random effects (a.k.a. features in ML jargon) design matrices of orders $n \times J_{k}^{\prime}$ and $n \times q_{j k}$, respectively. By assuming that the variable of interest follows the probability density function $f\left(y_{i} \mid \theta^{i}\right) \in \mathscr{D}$, a parametric family of distributions (see Table 1 in Rigby and Stasinopoulos (2005)) with $\theta^{i}=\left(\theta_{i 1}, \theta_{i 2}, \ldots, \theta_{i p}\right)^{\top}$ being a vector of $p$ parameters associated to the explanatory variables and to random effects, 3 each distribution parameter of the GAMLSS model can be written as a function of regressors.

In GAMLSS statistical models the $k$ th parameter $\theta_{k}$ is related to an additive predictor $\eta_{k}$ through input features and random effects via

$$
g_{k}\left(\theta_{k}\right)=\eta_{k}=\mathbf{X}_{k} \beta_{k}+\sum_{j=1}^{J_{k}} \mathbf{Z}_{j k} \gamma_{j k},
$$

where $g_{k}(\cdot)$ is a strictly monotonic link function, $\theta_{k}$ and $\eta_{k}$ are $n \times 1$ vectors, such as $\theta_{k}=\left(\theta_{1 k}, \theta_{2 k}, \ldots, \theta_{n k}\right)^{\top}, \beta_{k}=\left(\beta_{1 k}, \beta_{2 k}, \ldots, \beta_{J_{k}^{\prime} k}\right)^{\top}$ is a vector of parameters of length $J_{k}^{\prime}$ and $\gamma_{j k}$ is a $q_{j k}$-dimensional random variable which follows the $N_{q_{j k}}\left(0, G_{j k}^{-1}\right)$ law, where $G_{j k}^{-1}$ is the inverse of a symmetrical matrix $G_{j k}=G_{j k}\left(\lambda_{j k}\right)$ of size $q_{j k} \times q_{j k}$ which depends on a $\lambda_{j k}$ hyperparameter vector. If $G_{j k}$ is singular, then $\gamma_{j k}$ has a density function proportional to $\exp \left(-\frac{1}{2} \gamma_{j k}^{\top} G_{j k} \gamma_{j k}\right)$. A GAMLSS model can be expressed differently by including ML procedures in order to boost its predictive power. For example, if $Z_{j k}=I_{n}$, where $I_{n}$ is the identity matrix of type $n \times n$, and $\gamma_{j k}=h_{j k}=h_{j k}\left(x_{j k}\right)$ for all combinations of $j$ and $k$ expressed in equation 1 , then the GAMLSS model adopts a semi-parametric additive term:

$$
g_{k}\left(\theta_{k}\right)=\eta_{k}=X_{k} \beta_{k}+\sum_{j=1}^{j_{k}} h_{j k}\left(x_{j k}\right),
$$

where function $h_{j k}$ is an unknown function of the independent variable $x_{j k}$ and $h_{j k}\left(x_{j k}\right)$ is a vector that evaluates function $h_{j k}$ in $x_{j k}$. Furthermore, smoothers such as cubic splines, penalised splines, fractional polynomials, LOESS curves, terms of variable coefficients, neural networks, kernels, etc, can be included to deal with non-linearity, volatility structural changes and other particularities in the data (Wood et al. (2016); Wood (2017)).

The vector of fixed and/or random-effect parameters are estimated within the GAMLSS framework by maximising the penalised log-likelihood and this can be accomplished by using fast backfitting algorithms and resampling procedures (Rigby and Stasinopoulos (2005); Groll et al. (2019); Mayr et al. (2012)). Model selection is performed by finding the lowest global deviance GD $=-2 \ell(\hat{\theta})$, where $\ell(\hat{\theta})$ is the penalised log-likelihood. For the analysis of residuals, the normalised (randomised) quantile residuals plot can be used (Dunn and Smyth (1996)). Additionally, GAMLSS allows examining residuals via probability plots such as the worm plot (Buuren and Fredriks (2001)) which is an instrumental graphical technique for assessing the overall adequacy of the fitted model (see Stasinopoulos et al. (2017); Fasiolo et al. (2020)).

By using family of sets notation, a GAMLSS model can be represented for ML implementation as

$$
\mathscr{M}=\{\mathscr{D}, \mathscr{G}, \mathscr{T}, \lambda\}
$$

3 Generally, for application purposes $\max \{p\}=4$. Therefore, the first parameter is location (usually the mean or median), the second is scale (usually the standard deviation or precision), and the others are shape parameters (e.g. skewness and kurtosis). These are usually denoted by $\mu_{i}, \sigma_{i}, v_{i}, \tau_{i}$ for $i=1, \ldots, n$. For computational implementations of GAMLSS it is desirable that the probability density of $y$ and its first derivatives with respect to each of the parameters must be computable. Also, when the covariates are stochastic (useful in ML for investigating causal relation between variables) the density $f\left(y_{i} \mid \theta^{i}\right)$ is taken to be conditional on their values. 
where $\mathscr{D}$ represents a family of distributions, $\mathscr{G}$ specifies the set of link functions $\left(g_{1}, \ldots, g_{p}\right)$ for parameters $\left(\theta_{1}, \ldots, \theta_{p}\right), \mathscr{T}$ specifies the set of predictor terms $\left(t_{1}, \ldots, t_{p}\right)$ for predictors $\left(\eta_{1}, \ldots, \eta_{p}\right)$, and $\lambda$ specifies the set of hyperparameters. Thus, the linear regression model, for example, can be written as $y_{i} \sim \mathscr{D}\left(g_{1}(\mu(x)), g_{2}\left(\sigma(x)^{2}\right)\right)$ where $\mathscr{D}$ is the normal distribution, $\mathscr{G}=\left\{g_{1}, g_{2}\right\}=\{x, x\}$, and $\lambda=\left(\beta, \sigma^{2}\right)$. Note that in this case, $\mu(x)=x^{\top} \beta$ and $\sigma(x)^{2}=$ $\sigma^{2}$. Another important example is logistic regression or softmax regression in the context of neural networks. In such case, a GAMLSS model can be expressed as $y_{i} \sim \mathscr{D}\left(g_{1}(p(x))\right.$, where $\mathscr{D}$ is the Bernoulli distribution, $\mathscr{G}=\left\{g_{1}\right\}=\operatorname{logit}(x)=\log (x /(1-x))$ (logistic or softmax function), and $p(x)=P\left(y_{i}=1 \mid x_{i}\right)=\exp \left(x_{i}^{\top} \beta\right) /\left(1+\exp \left(x_{i}^{\top} \beta\right)\right)$ with $x^{\top} \beta$ being a linear predictor.

In order to compare two nested competing GAMLSS models $\mathscr{M}_{0}$ and $\mathscr{M}_{1}$ based on equation (3), that is, when one model can be obtained from the others by imposing parametric restrictions, the global deviance or a LASSO approach (Groll et al. (2019)) can be used to penalise overfittings and select the best model. When comparing two non-nested GAMLSS models (including models with smoothing terms (Hastie and Tibshirani $(\overline{1990)})$ ), the generalised Akaike information criterion (Akaike (1974)) and the $J$ and $M J$ tests (Davidson and MacKinnon, 1981, Godfrey, 2011; McAleer, 1995) can be used (Cribari-Neto and Lucena (2017)).

In a nutshell, GAMLSS is a framework that uses state-of-the-art algorithms for the modelling of continuous responses. As shown above, distributional regression analyses within a GAMLSS framework permit smooth alignment with well-known methods in ML. A final aspect to reiterate is that GAMLSS is designed to be a flexible and interpretable regressionbased method for statistical learning. This is a beneficial feature to counter "black-box" modelling and instead facilitate models' explainability and applicability (see Yu and Kumbier (2020)). For more details on GAMLSS see Stasinopoulos et al. (2017) and Rigby et al. (2020).

\section{The OULAD dataset}

The goal of this article is to showcase some of the statistical modelling capabilities of GAMLSS through a data set from the field of LA/EDM. The Open University Learning Dataset (OULAD; Kuzilek et al. (2017)) is an open access data set of about 32,593 students in a distance learning setting that relies on a Virtual Learning Environment (VLE). The OULAD has been released by the Open University; the largest distance learning institution in the United Kingdom with more than 165,000 students and hundreds of courses. Regular courses take approximately nine months to study and consist of multiple assignments and a final exam. The assignments can be divided into various categories being the Tutor Marked Assignments (TMAs) the most important as it represents key milestones in a study's schedule. The university employs a Moodle-like online system to deliver the course content to the students. This allows capturing valuable information such as students' demographics, study results and their behaviour within the VLE represented by the summaries of click-stream data.

One particular STEM course has been selected for the present analysis; FFF and its presentation (semester) $2013 \mathrm{~J}$ studied by 2,283 students. The course schedule is represented in Figure 1. The course contains five TMAs that represent the milestones for the topic learning periods. TMAs occur in weeks 2, 6, 13, 18 and 24 and at the end of the course an exam is taken. The exam takes place around four or more weeks have passed (in the current data set such information is missing). The present GAMLSS analysis focuses on this last TMA 
Table 1: List of independent variables ( ${ }^{\dagger}$ The complete list of regions can be found at https: //bit.1y/3kKF1zs)

\begin{tabular}{|c|c|}
\hline Attribute & Description \\
\hline gender & Student gender \\
\hline region & UK region, in which student lives ${ }^{\dagger}$ \\
\hline highest_education & The highest achieved education of the student \\
\hline imd_band & $\begin{array}{l}\text { percentile of the Index of Multiple Deprivation see Noble et al. } \\
\text { (2019) for details }\end{array}$ \\
\hline age_band & Student age band \\
\hline num_of_prev_attempts & $\begin{array}{l}\text { Indicator whether the student attempted the course in previous } \\
\text { years }\end{array}$ \\
\hline studied_credits & $\begin{array}{l}\text { Credits studied in parallel by student, serves as the estimation } \\
\text { of student workload }\end{array}$ \\
\hline disability & Indicator if student have disability \\
\hline cumulative_assessment_results & $\begin{array}{l}\text { Weighted sum of all previous TMAs: } a_{\text {sum }}=\sum_{n=1}^{4} w_{n} a_{n} \text {, } \\
\text { where } \vec{w}^{T}=(0.125,0.125,0.25,0.25) \text { is vector of correspond- } \\
\text { ing weights }\end{array}$ \\
\hline clicks_forumng & Sum of all clicks/actions student did in the discussion forum \\
\hline clicks_homepage & Sum of all clicks on course homepage \\
\hline clicks_oucontent & Sum of all views/clicks on TMAs assignments \\
\hline clicks_quiz & Sum of all clicks/attempts on non-graded quizzes \\
\hline clicks_subpage & Sum of all clicks when browsing the course web-page \\
\hline
\end{tabular}

(TMA 5) (in the data set it is labelled assessment_score). TMA 5 is thus the dependent variable and it ranges between 0 to 100 , such that values over 40 are considered as pass.

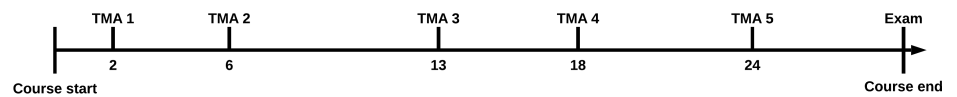

Fig. 1: Course schedule

The following groups of students were excluded from the data set: actively withdrawn students $(n=675)$ and students who did not submit all TMAs $(n=500)$. Actively withdrawn students were unregistered from the course before its end, and their information regarding VLE activities and assessments is incomplete. The second group did not submit all the assignments in time as required by the course. The resulting data set thus contains data of 1108 students. Table 1 lists and describes the independent variables in the data set. The first column contains the name of the variables and the second column shows a brief description of each variable (more details as to the source data set can be found in Kuzilek et al.(2017)). The click-stream information (i.e. "clicks_xy" variables) has been computed for the top five most common activity types in the VLE, and they represent $95 \%$ of all student click-stream data. 


\section{Statistical learning of the OULAD data set via GAMLSS}

The goal of the following modelling is to illustrate how GAMLSS can be used in practice and has no attempt at making theoretical LA/EDM-related claims based on the OULA data set. The first step in GAMLSS modelling is to find an appropriate marginal distribution (i.e. when the dependent variable is not condition on any covariates) that approximates well the observed values. The dependent variable was linearly transformed so that its values resided in the $[0,1]$ interval; i.e. FAS = assessment score/100; where 100 is the maximum assessment score.

GAMLSS enables to fit several distributions to the target variable via the histDist () and fitDist () functions. Given that the marginal distribution is bounded in the $[0,1]$ interval, the extra arguments type="real0to1" or type="realline" in fitDist() can be used to exhaustively search for distributions. The output of the search returns global deviance, AIC, and BIC values that assist in spotting candidate distributions. To avoid numerical problems, zeros and ones were converted to $0.5 / 100$ and to $99.5 / 100$ respectively (see Douma and Weedon (2019)). Note that choosing the distribution, or a set of candidate distributions, is not only a matter of statistical fitness but also of practical interpretability. Distributions with three or more parameters will tend to fit the skewness and kurtosis of the distribution better than distributions with two or less parameters. However, the applied researcher should prioritise distributions that parsimoniously explain changes in the values of the dependent variable in relation to the covariates in the context of the topic of the research. Figure 2 shows the AIC measures of several distributions fitted to the marginal FAS distribution (GAMLSS has several distributions available in the gamlss. dist package (loaded by default with the gamlss package)).

The probability density (PDF) and empirical cumulative distribution function (ECDF) plots indicate a negative skewness in FAS (see 3a). It is evident from the CDF plot that the Normal (NO) and Simplex (SIMPLEX) distributions provide poor fits even though these distributions are encountered in practical work (Rigby et al. 2020). The Beta class distributions (BE, BEINF, etc) are natural candidates (Ospina and Ferrari 2010) and exhibit reasonable behaviour. On the other hand, the generalised Beta type 1 (GB1) and skew $t$-type 2 distributions (ST2) (Rigby et al. 2020, Azzalini and Capitanio, 2003) gave the best fits.

Figure 4 shows FAS' PDFs conditioned on the covariates gender, disability and highest education. These PDF plots allow noticing differences in the location, dispersion and shape of the conditional distribution of FAS (i.e. for each combination of covariates). For example, it is evident that the higher the educational qualification, the higher the FAS and that students with disability tend to have lower FASs than non-disability students. Therefore, a useful approach to analyse the relation between FAS and its covariates is via GAMLSS as it allows to learn changes in the location, and other parameters, of the distribution of FAS as influenced by its covariates.

Using Wilkinson and Rogers' notation (Wilkinson and Rogers, 1973), the model to consider is $F A S \sim C+N$; where $F A S$ is the dependent variable (fractional assessment_score), $C$ represents a matrix with categorical covariates (gender, highest_education, age_band, disability, and type_of_click) and $N$ is a numeric covariate (number of clicks). For illustration purposes, fixed-effects-only regression models are specified, the set of distributions NNO, $\mathrm{BE}, \mathrm{ST} 2, \mathrm{~GB} 1\}$ is the response distribution space for the search and, the dispersion and shape parameters are assumed to be constant for all observations; that is, the focus is on the location parameter only. Note the Normal distribution (i.e. NO) was included for illustration purposes. The selection of the variables that compose the systematic dispersion submodel estimates is made by using the stepGAIC method in GAMLSS (Stasinopoulos et al. 2018). 


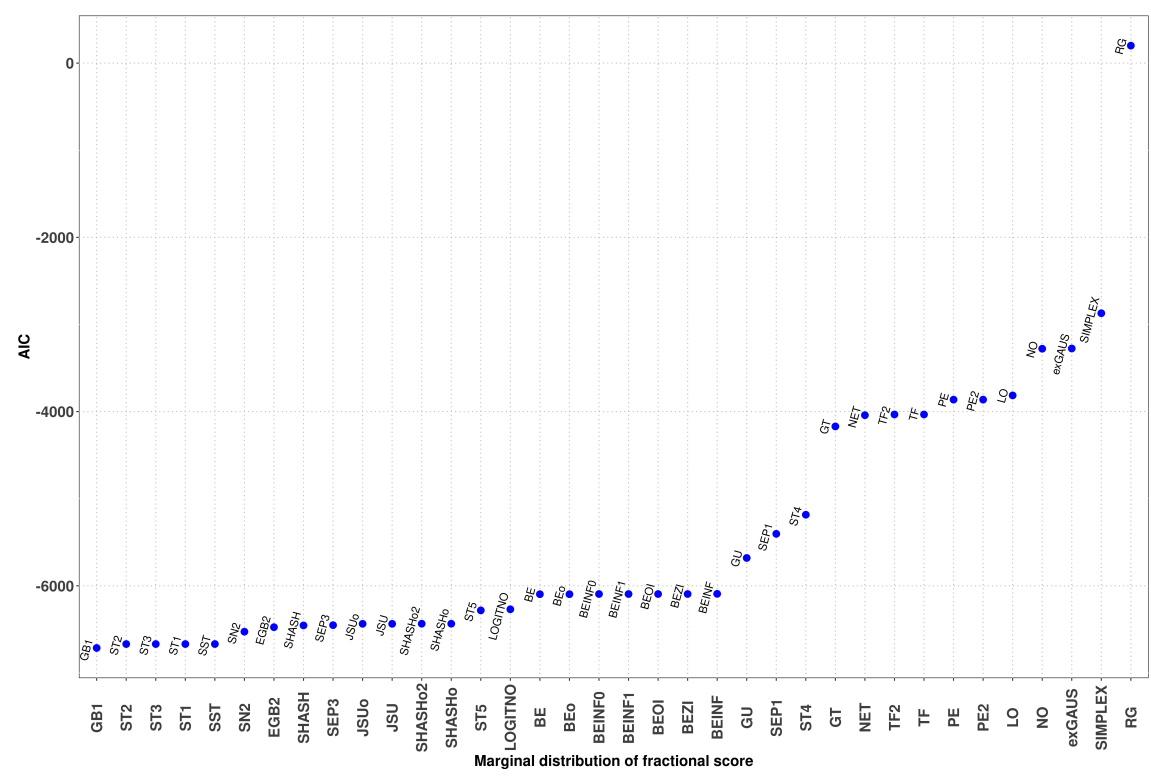

Fig. 2: AIC for the fitted models with different probability distributions. The lower the AIC value, the better the goodness-of-fit. For example, GB1 (generalised Beta type 1) to SST (skew $t$-distribution) are four-parameter distributions, while LO (logistic) to RG (Reverse Gumbel) are two-parameter distributions (except the exGAUS, ex-Gaussian distribution, which is a three-parameters distribution).

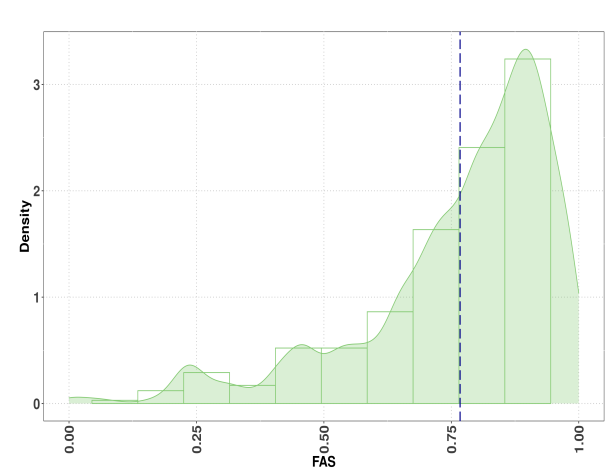

(a)

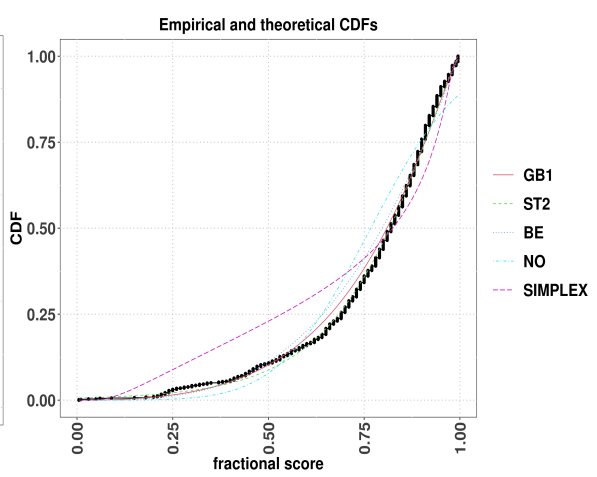

(b)

Fig. 3: FAS's PDF (a). FAS's empirical and theoretical CDFs (b). The vertical dotted line in the left plot indicates the variable's mean. The black line in the right plot shows the FAS's ECDF and the coloured lines represent five theoretical CDFs (ranked from best [generalised Beta type 1] to worst fit [Simplex]).

The selection of the distribution is based on the examination of quantile residuals via worm plots. 


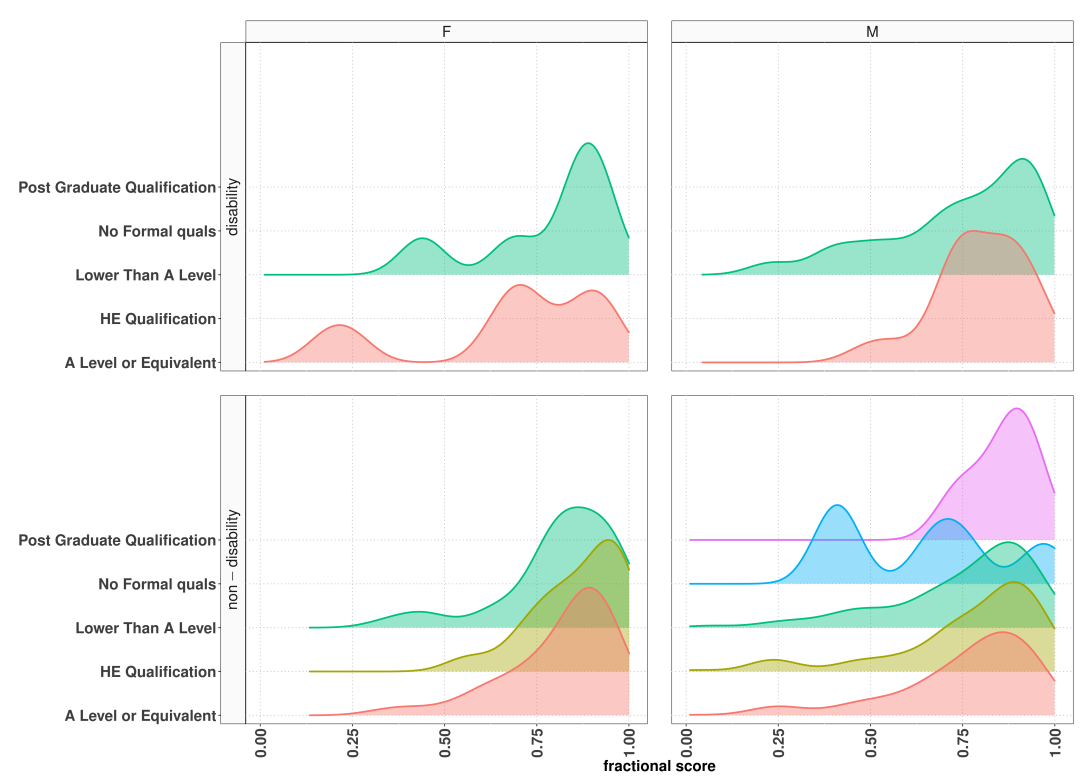

Fig. 4: FAS' PDF conditioned on the covariates gender (with two levels; F=females and $\mathrm{M}=$ males), disability (with two levels) and highest education (with five levels). The graph also indicates the data is imbalanced in that not all combinations of levels of the covariates have values. That is, while there are FAS values for males with non-disability at all education levels, there are FAS values for males with disabilities at two education levels only.

Table 2 presents the comparison of the fitted models with different distributions. As expected, the Normal distribution gave a poor fit. On the other hand, and as shown by the ECDF plots (see Figure 3b), the GB1 and ST2 distributions showed the best performance as indexed by the Likelihood, AIC and Degrees of Freedom estimates. The predictive power of the selected model is assessed via the pseudo- $R^{2}$ measure (via the GAMLSS R function Rsq ()). The pseudo- $R^{2}$ value (Cox and Snell, 1968, Nagelkerke et al., 1991) suggests that a model using the generalised Beta type 1 distribution gives the highest predictive power. Figure 5 shows a screenshot of the gamlss fit output based on the generic function summary. The results show the effects of the covariates on the location parameter only of the FAS distribution. The results indicate that all covariates have effects on the mean of the response variable FAS. For example, the output showed that, all other variables held constant, an increase in age is related to a decrease in FAS values.

Table 2: Goodness-of-fit measures after selection models

\begin{tabular}{lccccc}
\hline Model & Distribution & Likelihood & AIC & Degrees of Freedom & pseudo- $R^{2}$ \\
\hline Fit 1 & GB1 & -7025.59 & -6989.59 & 6234 & 0.111 \\
Fit 2 & ST2 & -6366.77 & -6332.77 & 6235 & 0.025 \\
Fit 3 & BE & -6342.56 & -6312.56 & 6237 & 0.097 \\
Fit 4 & NO & -3769.47 & -3739.47 & 6237 & 0.093 \\
\hline
\end{tabular}




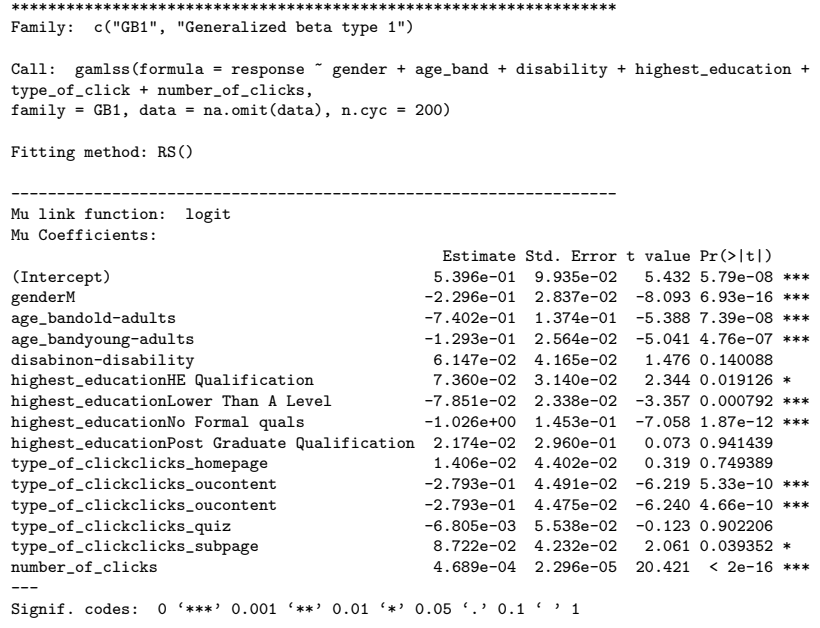

Fig. 5: Summary results of the best parametric model GB1 when modelling the location parameter $(\mu)$ only.

The adequacy of the fitted distributions is represented in Figure 6 via worm plots. A lack of fit is displayed by the residuals lying well above or below the value deviation 0.0. Also, the less closer the points of the plot are to the horizontal line at 0.0 , the more distant the distribution of the residuals is to a standard normal distribution. Additionally, a lack of fit is suggested when more than $5 \%$ of the points of the plot lie outside the two elliptic lines (those elliptic lines are point-wise $\approx 95 \%$ CIs). The fittings of the Beta, but particularly the Normal, distribution showed lack of fit based on the aspects described above and their inverted Ushape also signals the negative skewness in the residuals' distribution. This inverted U-shape also indicates that those distributions failed to correctly fit the high left-skewness of the data. Although the worm plot shapes of the GB1 and ST2 distributions suggest good fit, it was not perfect. Both struggled to fit the kurtosis of the marginal distribution and this is evidenced in the distribution of the residuals being leptokurtic in the case of the GB1 distribution (S-shape with left bent down) and platykurtic in the case of the ST2 distribution (S-shape with left bent up). Also, that some of the points in the plots representing the GB1 and ST2 distributions lay outside the $\approx 95 \%$ CIs indexes some degree of overdispersion in the data (see chapter 12 in Stasinopoulos et al. (2017) for details as to the interpretation of the worm plot).

So far all the GAMLSS modelling has been done only on the location parameter of the dependent distribution. A way to boost the predictive power is by also modelling the other parameters of the dependent variable. A likelihood ratio aimed at determining whether the GAMLSS scale and shape parameters were constant for all observations suggested these parameters were not constant. Thus, the covariates $\sim C+N$ were applied to the FAS's parameters $\sigma, v$ and $\tau$. As done above for the FAS's location parameter $\mu$, recursive covariate selection based on AIC was performed for the scale and shape parameters. The results showed that age not only affected FAS's average (as shown above) but also its dispersion (i.e. $\sigma$ ). The shape parameter $v$ was affected by age, highest education and the number of clicks. The parameter $\tau$ was only affected by disability and highest education. This new model had a pseudo- $R^{2}$ of 0.13 . That is, there was $15 \%$ increase in performance improvement. Figure 


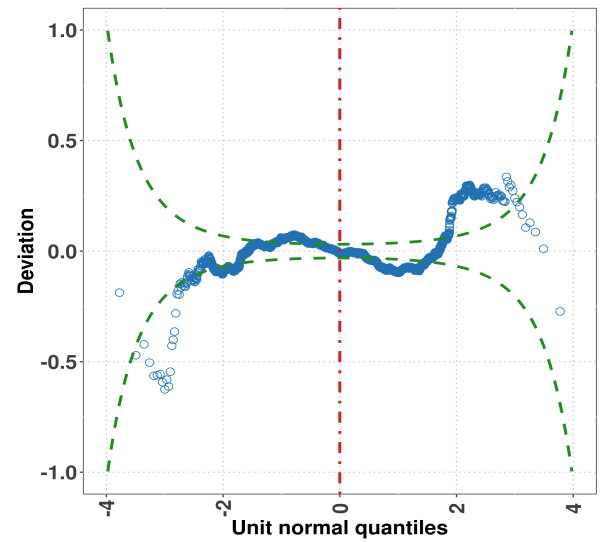

(a)

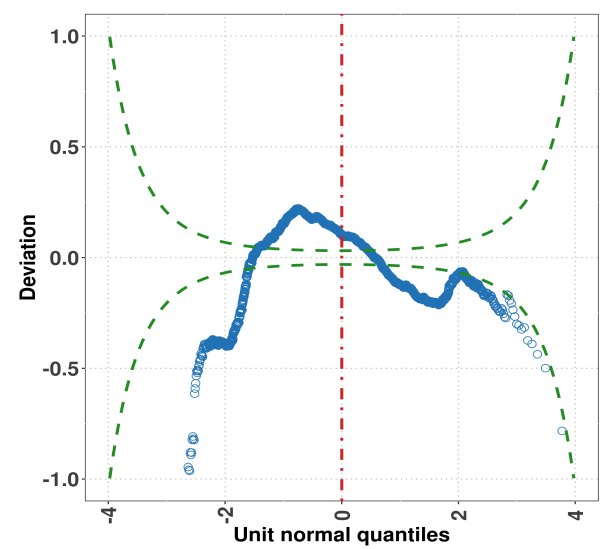

(c)

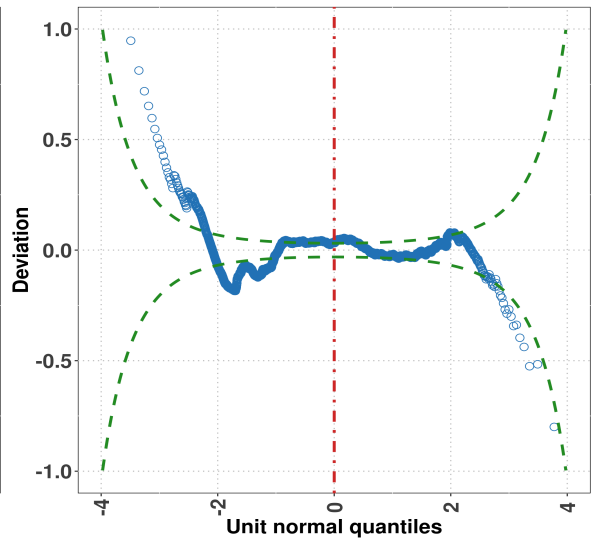

(b)

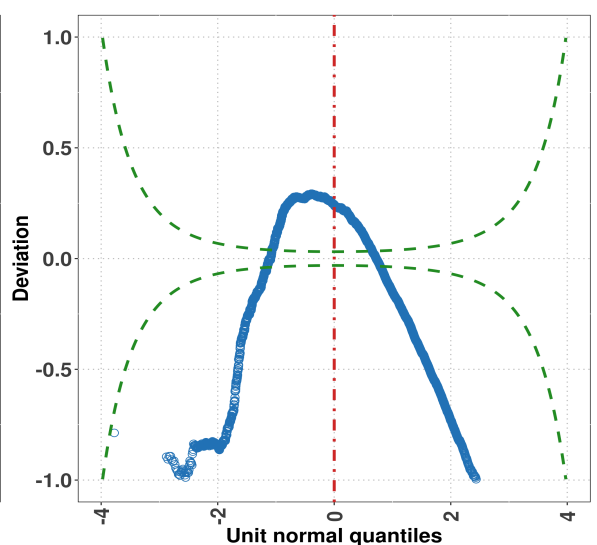

(d)

Fig. 6: Diagnostic worm plots for assessing the fitness of models using the generalised Beta type 1 (GB1) distribution (a), Skew $t$-distribution type 2 (ST2) (b), Beta (BE) distribution (c) and Normal (NO) distribution (d) to the FAS variable. A good fit is represented by $\approx 95 \%$ of values lying between the two green dotted elliptic lines and close to the deviation value of 0.0. In this example, the GB1 and ST2 distributions fit well most of the data but they struggle to fit the values in the tails of the FAS variable (although the ST2 distribution models better the right tail of the data than the GB1 distribution). However, compared to the GB1 and ST2 models, BE and NO exhibit a poor fit overall.

7 displays the residual worm plot for this comprehensive model. This new model indicated that fitting all the parameters of the FAS's distribution led to minimising the leptokurtosis in the residuals evident in Figure 6a, i.e. the points in the left tail of the worm plot are now closer to the $\approx 95 \%$ boundaries.

GAMLSS allows using complementary techniques to improve the modelling of the data but it would be prohibitive to attempt to cover them all herein. Thus, some techniques are briefly commented on. Variable selection can be carried out via cross-validation or LASSO 


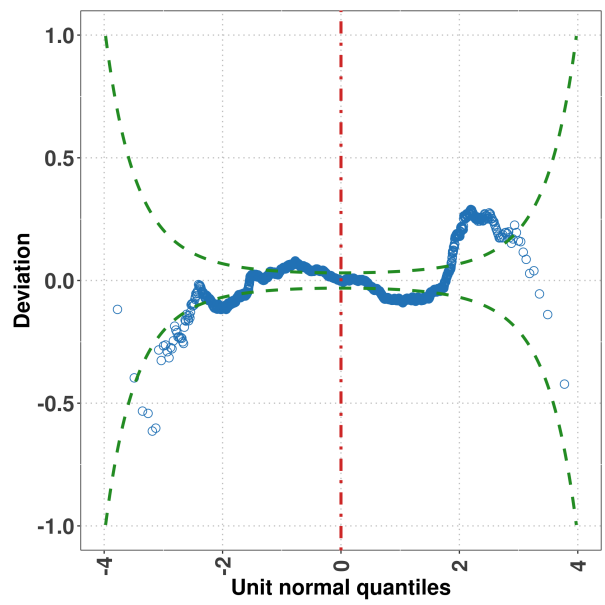

Fig. 7: Worm plot for the GB1 model when the $\mu, \sigma, v$ and $\tau$ parameters were modelled.

in order to control over-fitting by considering different link functions for the covariates (e.g. identity, inverse, reciprocal, etc). Also, the number of levels in categorical covariates can be reduced in order to improve the fitness of the model (see pcat () function in GAMLSS). GAMLSS also permits to robustify the model's fitness by countering the influence of outliers (via the function gamlssRobust). GAMLSS allows adding smoothing functions for numeric covariates in order to augment the prediction power; some of those functions available are cubic splines, locally weighted regression, penalised splines, and neural networks. The modelling performed here was fully parametric; so smoothers are to be used if a semiparametric modelling is sought.

Cross-validation is a ubiquitous step in ML. In GAMLSS, $k$-fold cross-validation is attained via the gamlssCV () function. If the goal is to fit a gamlss model to the training data set and estimate the validation global deviance for the validation data set, the gamlssVGD() function can be used. It is important to recall, though, that cross-validation requires relative stability of the structured data and complete observations for each level within each variable in order to obtain reliable estimates (Keevers, 2019, Wang and Gelman, 2015, Gronau and Wagenmakers, 2019). As shown in Figure 4, some levels within the education level variable had non-existent observations for males and females and their interaction with disability / non-disability; this situation thus led to estimation issues when cross-validation was used on this data. Missing values can be handled by creating predictive models that include imputation or LASSO-type regularisation (Arrieta et al. 2020, Hamzah et al. 2020).

\section{Discussion and conclusions}

This article had the goal of showcasing some of the modelling properties allowed by the GAMLSS framework. In order to do so, an open access data set pertinent to LA/EDM was used. The analyses did not intend to make claims about LA/EDM aspects relating to the data set but simply illustrate how GAMLSS could be used for a supervised statistical learning of data. It was then argued and showed that GAMLSS is a flexible and interpretable regression-oriented modelling approach that enables investigating the effect of the covariates 
on the dependent variable's location, scale, skewness and kurtosis parameters (more details on GAMLSS at https://www.gamlss.com/). The analysis also illustrated that GAMLSS allows building both explanatory and predictive models and producing both types of models is a must in proper statistical learning (see e.g. Shmueli (2010); Yarkoni and Westfall (2017)). The following paragraphs revolve around methodological and statistical issues relating to GAMLSS-type analyses and statistical learning in general.

The data was modelled with a generalised Beta type 1 distribution (GB1). Although the traditional two-parameters Beta distribution (BE) did not provide a good fit, it does not entail it is disposable. It may be well the case that the GB1 may not be a good model in similar data set, while the BE provides a better fit. A short document found in the repository (see link in the last paragraph) provides mathematical arguments in favour of the Beta distribution. It is also worth mentioning that GAMLSS is not the only way to analyse continuous data. As shown in the supplementary materials, a GAM (with penalisation) analysis is also possible. GAM-type analysis is contained within the GAMLSS framework and it has been shown to be instrumental in modelling auto-correlations in experimental data (Baayen et al. (2017)). Alternatively, quantile regression and robust regression could have been educated choices. Performing several valid statistical analyses is indeed encouraged as they enable to determine patterns in data; this approach is known as multiverse analysis (Steegen et al. (2016)).

Although it was shown herein that a GAMLSS framework can cater for a supervised statistical learning approach to data analysis, it does not prevent mixing GAMLSS with unsupervised learning techniques. For example, classification algorithms such as the "one rule" (a.k.a. 1R, see Holte (1993). Implemented in R via the OneR R package) and "Boruta" (Kursa and Rudnicki (2010). Implemented in $\mathrm{R}$ via the Boruta $\mathrm{R}$ package) can be used for variable selection and candidate GAMLSS regression models can be built by combining the best subset of variables. Finally, the resulting models' predictive power could be assessed via cross-validation. Indeed, there is a recent method called distributional regression that blends decision trees (a predictive model popular in ML) and GAMLSS regression (see Schlosser et al. (2019) and the disttree R package). These are approaches worth exploring in silico and through real datasets. Ultimately, the goal is to promote statistical learning and modelling and minimise reliance on null hypothesis testing. GAMLSS, and the techniques mentioned above, allow precisely this.

Finally, there is a recent interest in the topic of causality. There are recent arguments indicating that regression techniques are tools "geared towards causality" in that they can be used to examine stabilisation of estimated fits across perturbations (Bühlmann (2020a)). This is a very important task in that stability and invariance of regression fits is a sensible proxy of causality. GAMLSS is very flexible regression tool that can be used for tackling causality. Having said this, future work should put this claim to the test.

The $\mathrm{R}$ codes and data sets used in the analyses can be found at https://figshare. com/projects/GAMLSS_for_educational_data_mining_and_learning_analytics/ 91199. A couple of brief manuscripts relating to GAM modelling and the importance of the Beta distribution are also found therein.

Acknowledgements J. K. was supported by a junior grant research project by Czech Science Foundation GACR no. GJ18-04150Y and also partially by the long-term strategic development financing of the Institute of Computer Science (Czech Republic RVO 67985807). M. T. acknowledges support from Research Regular Projects 2019, Universidad Tecnológica Metropolitana (code LPR19-02). J. G. acknowledges partial support from Fondecyt Grant 1201129. R. O. thanks partial support by the National Council for Scientific and Technological Development (CNPq) in Brazil, through the grant no. 305305/2019-0. F. M-R. thanks Paul Bühlmann for clarifying aspects in relation to causality and regression models. 


\section{Conflict of interest and CRediT}

F.M-R., M.T., and M.B.: conceptualisation, methodology, software | R.O., M.B., and F.MR.: statistical analyses | J.K.: data curation | all authors: writing-reviewing and editing. On behalf of all authors, the corresponding author states that there is no conflict of interest.

\section{References}

Akaike H (1974) A new look at the statistical model identification. IEEE transactions on automatic control 19(6):716-723

Akantziliotou K, Rigby R, Stasinopoulos D (2002) The R implementation of generalized additive models for location, scale and shape. In: Statistical modelling in Society: Proceedings of the 17th International Workshop on statistical modelling, Statistical Modelling Society, pp 75-83

Arrieta AB, Díaz-Rodríguez N, Del Ser J, Bennetot A, Tabik S, Barbado A, García S, GilLópez S, Molina D, Benjamins R, et al. (2020) Explainable artificial intelligence (xai): Concepts, taxonomies, opportunities and challenges toward responsible ai. Information Fusion 58:82-115

Azzalini A, Capitanio A (2003) Distributions generated by perturbation of symmetry with emphasis on a multivariate skew t-distribution. Journal of the Royal Statistical Society: Series B (Statistical Methodology) 65(2):367-389

Baayen H, Vasishth S, Kliegl R, Bates D (2017) The cave of shadows: Addressing the human factor with generalized additive mixed models. Journal of Memory and Language 94:206234

Barndorff-Nielsen OE (1980) Exponential families. Wiley Online Library

Bates S, Sesia M, Sabatti C, Candès E (2020) Causal inference in genetic trio studies. Proceedings of the National Academy of Sciences 117(39):24117-24126, DOI 10.1073/pnas.2007743117

Bennette C, Vickers A (2012) Against quantiles: categorisation of continuous variables in epidemiological research, and its discontents. BMC Medical Research Methodology 12(21), DOI doi.org/10.1186/1471-2288-12-21

Bono R, Blanca M, Arnau J, Gómez-Benito J (2017) Non-normal distribution commonly used in health, education, and social sciences. A systematic review. Frontiers in Psychology 8(1602), DOI 10.3389/fpsyg.2017.01602

Breiman L (2001) Statistical modelling: The two cultures. Statistical Science 16(3):199-231

Bühlmann P (2020a) Invariance, causality and robustness. Statistical Science 35(3):404-426

Bühlmann P (2020b) Toward causality and improving external validity. Proceedings of the National Academy of Sciences 117(42):25963-25965, DOI 10.1073/pnas.2018002117

Buuren Sv, Fredriks M (2001) Worm plot: a simple diagnostic device for modelling growth reference curves. Statistics in Medicine 20(8):1259-1277

Casella G, Berger RL (2002) Statistical inference, vol 2. Duxbury Pacific Grove, CA

Cole TJ, Green PJ (1992) Smoothing reference centile curves: the lms method and penalized likelihood. Statistics in medicine 11(10):1305-1319

Cox DR, Snell EJ (1968) A general definition of residuals. Journal of the Royal Statistical Society: Series B (Methodological) 30(2):248-265

Cribari-Neto F, Lucena SE (2017) Non-nested hypothesis testing inference for gamlss models. Journal of Statistical Computation and Simulation 87(6):1189-1205 
Davidson R, MacKinnon JG (1981) Several tests for model specification in the presence of alternative hypotheses. Econometrica: Journal of the Econometric Society pp 781-793

De Bastiani F, Rigby RA, Stasinopoulous DM, Cysneiros AH, Uribe-Opazo MA (2018) Gaussian markov random field spatial models in gamlss. Journal of Applied Statistics 45(1):168-186

De Castro M, Cancho VG, Rodrigues J (2010) A hands-on approach for fitting longterm survival models under the gamlss framework. Computer Methods and Programs in Biomedicine 97(2):168-177

Douma JC, Weedon JT (2019) Analysing continuous proportions in ecology and evolution: A practical introduction to beta and dirichlet regression. Methods in Ecology and Evolution 10(9):1412-1430

Dunn PK, Smyth GK (1996) Randomized quantile residuals. Journal of Computational and Graphical Statistics 5(3):236-244

Fasiolo M, Nedellec R, Goude Y, Wood SN (2020) Scalable visualization methods for modern generalized additive models. Journal of computational and Graphical Statistics 29(1):78-86

Florencio L, Cribari-Neto F, Ospina R (2012) Real estate appraisal of land lots using GAMLSS models. Chilean Journal of Statistics 3(1):75-91

Friedman JH, Silverman BW (1989) Flexible parsimonious smoothing and additive modeling. Technometrics 31(1):3-21

Gelman A, Park D (2008) Splitting a predictor at the upper quarter or third and the lower quarter or third. The American Statistician 62(4):1-8

Gilchrist R, Kamara A, Rudge J (2009) An insurance type model for the health cost of cold housing: an application of gamlss. REVSTAT-Statistical Journal 7(1):55-66

Godfrey LG (2011) Robust non-nested testing for ordinary least squares regression when some of the regressors are lagged dependent variables. Oxford Bulletin of Economics and Statistics 73(5):651-668

Groll A, Hambuckers J, Kneib T, Umlauf N (2019) Lasso-type penalization in the framework of generalized additive models for location, scale and shape. Computational Statistics \& Data Analysis 140:59-73

Gronau QF, Wagenmakers EJ (2019) Limitations of Bayesian leave-one-out cross-validation for model selection. Computational Brain \& Behavior 2(1):1-11

Hamzah FB, MohdHamzah F, Razali SFM, Jaafar O, AbdulJamil N (2020) Imputation methods for recovering streamflow observation: A methodological review. Cogent Environmental Science 6(1):1745133

Hastie TJ, Tibshirani RJ (1990) Generalized additive models london chapman and hall. Inc

Hofner B, Mayr A, Schmid M (2014) gamboostlss: An R package for model building and variable selection in the gamlss framework. arXiv preprint arXiv:14071774

Holte RC (1993) Very simple classification rules perform well on most commonly used datasets. Machine Learning 11:63-91

$\mathrm{Hu}$ W, Swanson BA, Heller GZ (2015) A statistical method for the analysis of speech intelligibility tests. PloS one 10(7):e0132409

Imai K, King G, Stuart E (2008) Misunderstanding between experimentalists and observationalists about causal inference. Journal of the Royal Statistical Society, Series A 171(2):481-502

Joksimovic S, Poquet O, Kovanovic V, Dowell N, Mills C, Gasevic D, Dawson S, Graesser A, Brooks C (2018) How do we model learning at scale? a systematic review of research on MOOCs. Review of Educational Research 88(1):43-86 
Keevers TL (2019) Cross-validation is insufficient for model validation. Joint and Operations Analysis Division, Defence Science and Technology Group: Victoria, Australia

Kursa M, Rudnicki WR (2010) Feature selection with the boruta package. Journal of Statistical Software, Articles 36(11):1-13, DOI 10.18637/jss.v036.i11, URL https: //www.jstatsoft.org/v036/i11

Kuzilek J, Hlosta M, Zdrahal Z (2017) Open University Learning Analytics dataset. Scientific Data 4:170171, URL http://dx.doi.org/10.1038/sdata.2017.171http: $/ / 10.0 .4 .14 /$ sdata.2017.171

Van der Laan M (2017) Targeted learning: the link from statistics to data science. STAtOR 18(4):12-16

Mayr A, Fenske N, Hofner B, Kneib T, Schmid M (2012) Generalized additive models for location, scale and shape for high dimensional data-a flexible approach based on boosting. Journal of the Royal Statistical Society: Series C (Applied Statistics) 61(3):403427

McAleer M (1995) The significance of testing empirical non-nested models. Journal of Econometrics 67(1):149-171

McCullagh P (2002) What is a statistical model? The Annals of Statistics 30(5):1225-1310

McCulloch CE (2000) Generalized linear models. Journal of the American Statistical Association 95(452):1320-1324

Motz B, Carvalho P, de Leeuw J, Goldstone R (2018) Embedding experiments: staking causal inference in authentic educational contexts. Journal of Learning Analytics 5(2)

Nagelkerke NJ, et al. (1991) A note on a general definition of the coefficient of determination. Biometrika 78(3):691-692

Nelder JA, Wedderburn RW (1972) Generalized linear models. Journal of the Royal Statistical Society: Series A (General) 135(3):370-384

Noble S, McLennan D, Plunkett E, Gutacker N, Silk M, Wright G (2019) Ministry of Housing, Communities and Local Government. https://assets.publishing. service.gov.uk/government/uploads/system/uploads/attachment_data/ file/833947/IoD2019_Research_Report.pdf

Ospina R, Ferrari SL (2010) Inflated beta distributions. Statistical Papers 51(1):111

Picard RR, Cook RD (1984) Cross-validation of regression models. Journal of the American Statistical Association 79(387):575-583

Rigby MD R Aand Stasinopoulos, Heller GZ, de Bastiani F (2020) Distributions for modeling location, scale, and shape using GAMLSS in R. CRC Press

Rigby R, Stasinopoulos D (1996) A semi-parametric additive model for variance heterogeneity. Statistics and Computing 6(1):57-65

Rigby R, Stasinopoulos D (2001) The gamlss project: a flexible approach to statistical modelling. In: New trends in statistical modelling: Proceedings of the 16th international workshop on statistical modelling, University of Southern Denmark, vol 337, p 345

Rigby RA, Stasinopoulos MD (2005) Generalized additive models for location, scale and shape. Journal of the Royal Statistical Society: Series C (Applied Statistics) 54(3):507554

Romero C, Ventura S (2020) Educational data mining and learning analytics: an updated survey. WIRES Data Mining and Knowledge Discovery 10(e1355), DOI doi.org/10.1002/ widm. 1355

Schlosser L, Hothorn T, Stauffer R, Zeileis A (2019) Distributional regression forests for probabilistic precipitation forecasting in complex terrain. Annals of Applied Statistics 13(3):1564-1589

Shmueli G (2010) To explain or to predict? Statistical Science 25(3):289-310 
Smith A, Hofner B, Lamb JS, Osenkowski J, Allison T, Sadoti G, McWilliams SR, Paton P (2019) Modeling spatiotemporal abundance of mobile wildlife in highly variable environments using boosted gamlss hurdle models. Ecology and evolution 9(5):2346-2364

Stasinopoulos MD, Rigby RA, et al. (2007) Generalized additive models for location scale and shape (gamlss) in r. Journal of Statistical Software 23(7):1-46

Stasinopoulos MD, Rigby RA, Heller GZ, Voudouris V, de Bastiani F (2017) Flexible regression and smoothing using GAMLSS in R. CRC Press

Stasinopoulos MD, Rigby RA, de Bastiani F (2018) GAMLSS: A distributional regression approach. Statistical Modelling 18(3-4):248-273

Steegen S, Tuerlinckx F, Gelman A, Vanpaemel W (2016) Increasing transparency through a multiverse analysis. Perspectives in Psychological Science 11(5):702-712

Thomas J, Mayr A, Bischl B, Schmid M, Smith A, Hofner B (2018) Gradient boosting for distributional regression: faster tuning and improved variable selection via noncyclical updates. Statistics and Computing 28(3):673-687

Umlauf N, Klein N, Zeileis A (2018) Bamlss: Bayesian additive models for location, scale, and shape (and beyond). Journal of Computational and Graphical Statistics 27(3):612627

Wang W, Gelman A (2015) Difficulty of selecting among multilevel models using predictive accuracy. Statistics and Its Interface 8(2):153-160

Wilkinson GN, Rogers CE (1973) Symbolic description of factorial models for analysis of variance. Journal of the Royal Statistical Society Series C (Applied Statistics) 22(3):392399

Wood SN (2017) Generalized Additive Models: An Introduction with R, 2nd ed. Chapman $\&$ Hall, CRC.

Wood SN, Pya N, Saefken B (2016) Smoothing parameter and model selection for general smooth models (with discussion). JASA 111(516):1548—-1575

Yarkoni T, Westfall J (2017) Choosing prediction over explanation in psychology: Lessons from machine learning. Perspectives on Psychological Science 12(6):1100-1122

Yu B, Kumbier K (2020) Veridical data science. Proceedings of the National Academy of Sciences of the United States of America 117(8):3920-3929 